\title{
The effect of personalized nursing intervention based on CGA on the quality of life for elderly patients with chronic diseases.
}

\author{
Fen Gong ${ }^{1}$, Yan-ling $\mathrm{Hu}^{2 *}$ \\ ${ }^{1}$ Department of Surgical Outpatient, Jining No.1 People's Hospital, Jining City, Shandong Province, PR China \\ ${ }^{2}$ Department of Basic Medicine, Chongqing Three Gorges Medical College, Wanzhou District, Chongqing City, PR \\ China
}

\begin{abstract}
Objective: To explore the effect and clinical value of personalized nursing intervention according to Comprehensive Geriatric Assessment (CGA) on the quality of life in elderly patients with chronic diseases.

Methods: A total of $\mathbf{3 0 0}$ elderly patients with chronic diseases were randomly divided into control group $(n=150)$ and observation group $(n=150)$. The patients in the control group were treated with routine nursing. On the basis of routine nursing, the observation group was treated with personalized nursing intervention based on CGA. Before intervention and at six months after discharged, two groups were assessed by using the quality of life scale, and their effect on quality of life and satisfaction rate of treatment and nursing work were compared before and after the intervention.

Results: After the intervention, the scores in the elderly patients with the observation group in physiology, psychology, independence, social relations, environment, and general quality of life were higher than those of the control group $(t=4.998,4.937,7.174,5.106,4.087$, all $P<0.05)$, and differences were statistically significant, and nursing satisfaction at six months after discharged were significantly higher than that of the control group $\left(\chi^{2}=5.556, P<0.05\right)$.

Conclusion: Personalized nursing intervention based on CGA can improve the nursing of elderly patients with chronic diseases and improve the satisfaction of patient during hospitalization and nursing after discharged, then improve the quality of life of patients.
\end{abstract}

Keywords: Comprehensive geriatric assessment, Elder patients, Chronic diseases, Personalized nursing. Accepted on December 18, 2017

\section{Introduction}

With the increasing aging of the global population, elderly patients with chronic diseases are increasing year by year [1]. The elderly, due to cognitive impairment, reduced mobility, unstable movements, incontinence (more than $65 \mathrm{y}$ old) and other characteristics, seriously affect their quality of life, thus promoting the demand for medical services [2,3]. Active and effective nursing intervention for elderly patients with chronic diseases is helpful to improve the treatment effect and the quality of life $[4,5]$.

Comprehensive Geriatric Assessment (CGA) is the process of diagnosis and treatment of a multilatitude interdisciplinary, and the contents of evaluation mainly consist of health assessment, cognitive and psychological function, physical function assessment, social assessment and environmental assessment, to develop a comprehensive coordination treatment and rehabilitation plan and long-term follow-up plan for elderly patients, and to maximize the health of elderly people [6].
Personalized care comes from the holistic nursing mode of thinking development, regards patients as the integrity of the individual, the integration of the personality characteristics of medical care and the patients themselves, to provide the most suitable nursing service for patients [7]. The focus of personalized nursing is to improve the cognition of elderly patients on disease, reduce the pain of patients, improve the quality of life, and promote physical and mental health [8]. The study of individualized nursing comprehensive geriatric assessment based on the formulation of intervention on the quality of life of elderly patients with chronic diseases, seeking effective nursing measures can improve the quality of life in elderly patients with chronic diseases, the clinical nursing service quality and provide a theoretical basis for the clinical promotion of quality care services. 


\section{General Information}

\section{Research subjects}

Totally, 300 cases as the object of study from January 2014 to September 2016 from department of Respiratory Medicine, Cardiology, Hypertension Endocrinology, Oncology ward were collected in our hospital, inclusion criteria: 1. chronic diseases such as the high blood pressure, coronary heart disease, diabetes, chronic respiratory diseases, malignant tumors of the elderly patients, aged $60-85 \mathrm{y} ; 2$. conscious and normal communicators. Exclusive criteria: 1. incomplete clinical records or patients unable to communicate with each other; 2. patients with acute and severe diseases; 3. complete loss of living ability. Patients and their families voluntarily participated in and signed informed consent and the study was approved by the Ethics Committee of our institute. The subjects were randomly divided into control group and observation group, 150 cases in each group. There was no significant difference in gender, age, duration, complication and Body Mass Index (BMI) in the two groups $(\mathrm{P}<0.05$, Table $1)$.

Table 1. Comparison of two groups of patients with general information (mean $\pm S D$ ).

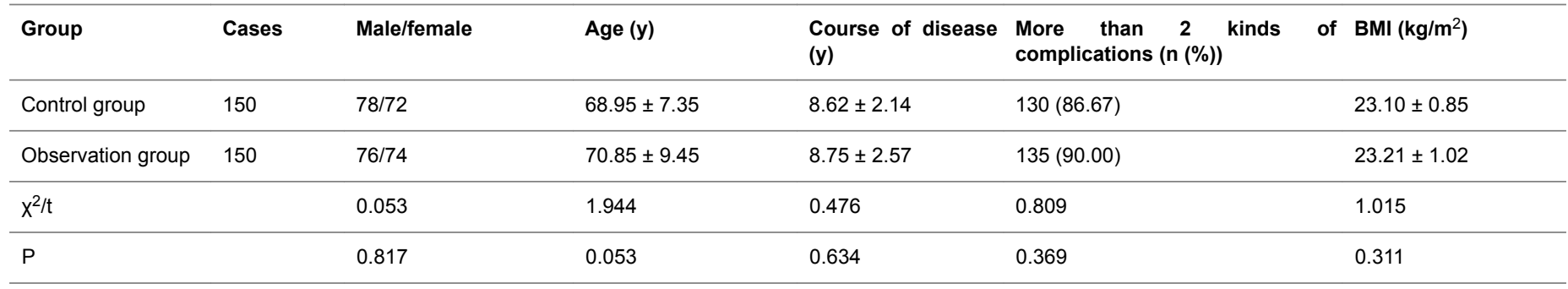

\section{Method}

Control group: The control group was given routine care, after admission, the nurses carried out health education to the patients and their families, to understand the causes of chronic diseases, treatment and precautions, to help patients establish a correct concept of life. Monitoring the patient's medication (such as antihypertensive drugs, hypoglycemic agents, lipidlowering drugs, etc.) and diet, to avoid spicy excitant food, light diet, etc. should also ensure the safety of patients to avoid falling down from bed or falling down and so on.

Observation group: The observation group: on the basis of the control group, the observation group were given personalized nursing intervention based on CGA. Routine medical assessment was carried out at admission, and CGA manual was filled in and CGA files were brought into the medical records. First, CGA positive patients were screened by 1-3 d after admission, and CGA labels were attached to medical records and nursing care, which caused medical staff to pay attention to them and needed to focus on key shift. Second, bringing the issues such as comprehensive care, cognitive ability, psychological status, physical function and nutrition assessed by CGA into the nursing plan. Third, pointing out the existing deficiencies and goals in nursing care according to positive problems that have been assessed. Forth, formulating intervention plan with the chief physician. Fifth, formulating the key intervention prescriptions for sleep disorders, cognitive disorders, constipation, chronic pain, malnutrition and other complications, giving the corresponding nursing measures, and personalized education, finding out potential problems timely, conducting the multidisciplinary consultation when necessary. Hospital referral cards were issued by nurses, the guidance before discharged and the care after discharged were offered by nurses. Follow up time: one week after discharged, the nurses followed up by telephone, 3 months after discharged by family visit, 6 months after discharged, the patients were sent back to the department for reexamination, in order to systematically understand the quality of life of patients.

Evaluation of elderly patients with chronic diseases in CGA manual mainly includes: (1) medical assessment: including medical history, medication management, vision, hearing, teeth, defecation and urination, sleep, falls, chronic pain and other issues. (2) cognitive function assessment: the Loewenstein Occupational Therapy Cognitive Assessment (LOTCA) scale [9] was used. (3) assessment of psychological function (emotional status): the elderly depression scale was used to assess [10]. (4) physical function assessment: the use of ability of daily life, instrumental daily living ability scale and fall risk assessment scale [11]. (5) assessment of nutritional risk: the Mini Nutritional Assessment scale (MNA) score was used [12]. (6) assessment of social, environmental and economic status, including economic situation, social support system, living environment, residential safety, etc.

\section{Evaluation of efficacy}

First, the quality of life: The World Health Organization Quality of Life Instruments (WHOQOL-100) is covering the physiology (pain and discomfort, energy and fatigue, sleep and rest), psychology (positive feelings, thoughts, learning, memory and attention, self-esteem, physical and appearance, negative feelings), independence (mobility, daily life, medical care and drug dependence, ability to work), social relationships (personal relationship, required social support satisfaction, sexual life) and environment (social security, housing environment, economic sources, medical services and social security, access to new information, knowledge and skills opportunities, recreation participation, environmental conditions, traffic conditions), and spiritual support/religious/ 
The effect of personalized nursing intervention based on CGA on the quality of life for elderly patients with chronic diseases

personal beliefs, the six dimensions and twenty-four aspects were assessed for evaluating quality of life. It is an effective tool to assess the quality of life of the elderly [13]. Elderly patients can score according to their own feelings in the past 2 $\mathrm{w}$, the scores are divided into satisfaction, basic satisfaction and dissatisfaction. The higher the score was, the better the quality of life was. The time of personalized nursing intervention can be chosen the day of admission and 6 months after discharged.

Second, satisfaction rate: the scores on nursing satisfaction according to the nursing staff's working attitude and serious degree, professional operation level, and their disease recovery, using percentile system, 80-100 points was divided into satisfaction, 60-80 points was regarded as the basic satisfaction, $<60$ points was not satisfied.

\section{Statistical analysis}

The software SPSS 21.0 was used to analyse all the data. The measurement data were expressed as mean \pm standard deviation, and analysed by t-test. The enumeration data were expressed as number or percentage of cases, and analysed by Chi-square test. $\mathrm{P}<0.05$ was considered as the critical criterion for statistical significance.

\section{Results}

\section{Comparison of quality of life between the two groups before intervention}

Before the intervention, there were no significant differences in the scores of the quality of life scale between the observation group and the control group ( $\mathrm{P}>0.05$, Table 2$)$.

\section{Comparison of quality of life before and after intervention in control group}

In the control group, there was no significant difference in the scores of quality of life scale before and after the intervention $(\mathrm{P}>0.0$, Table 3).

\section{Comparison of quality of life before and after intervention in observation group}

After intervention, the scores of the quality scale of the observation group were significantly higher than those before the intervention $(\mathrm{P}<0.05$, Table 4$)$.

\section{Comparison of satisfaction of treatment and nursing between the two groups after intervention}

After intervention, the elderly patients with the observation group were more satisfied with the nursing work than the control group in hospitalization and 6 months after discharged, and the difference was statistically significant $(\mathrm{P}<0.05$, Table 5).

Table 2. Comparison of scores of quality of life between the two groups before intervention (mean $\pm S D$ ).

\begin{tabular}{|c|c|c|c|c|}
\hline Items & $\begin{array}{l}\text { Observation } \\
\text { group }(n=150)\end{array}$ & $\begin{array}{l}\text { Control } \\
(n=150)\end{array}$ & $\mathbf{t}$ & $\mathbf{P}$ \\
\hline Physiology & $11.81 \pm 3.05$ & $12.03 \pm 2.58$ & 0.675 & 0.500 \\
\hline Psychology & $12.02 \pm 2.91$ & $12.29 \pm 2.14$ & 0.916 & 0.361 \\
\hline Independence & $11.10 \pm 2.92$ & $11.80 \pm 3.51$ & 1.878 & 0.061 \\
\hline Social relations & $11.41 \pm 2.63$ & $11.92 \pm 2.04$ & 1.877 & 0.062 \\
\hline Environment & $11.28 \pm 2.59$ & $11.58 \pm 1.92$ & 1.140 & 0.255 \\
\hline $\begin{array}{lr}\text { Spiritual } & \text { support/ } \\
\text { personal } & \text { belief/ } \\
\text { religion } & \end{array}$ & $10.67 \pm 2.41$ & $10.39 \pm 2.82$ & 0.925 & 0.356 \\
\hline $\begin{array}{l}\text { Overall quality of } \\
\text { life }\end{array}$ & $11.23 \pm 2.18$ & $11.26 \pm 1.93$ & 0.126 & 0.900 \\
\hline
\end{tabular}

Table 3. Comparison of life quality scores before and after intervention in the control group (mean $\pm S D)$.

\begin{tabular}{lllll}
\hline Items & $\begin{array}{l}\text { After } \\
\text { intervention }\end{array}$ & $\begin{array}{l}\text { Before } \\
\text { intervention }\end{array}$ & $\mathbf{t}$ & $\mathbf{P}$ \\
\hline Physiology & $12.68 \pm 3.28$ & $12.03 \pm 2.58$ & 1.937 & 0.054 \\
\hline Psychology & $12.84 \pm 2.91$ & $12.29 \pm 2.14$ & 1.865 & 0.063 \\
\hline $\begin{array}{l}\text { Independence } \\
\text { Social relations }\end{array}$ & $12.45 \pm 3.39$ & $11.80 \pm 3.51$ & 1.631 & 0.104 \\
\hline $\begin{array}{l}\text { Environment } \\
\text { Spiritual support/ }\end{array}$ & $10.83 \pm 2.97$ & $10.39 \pm 2.82$ & 1.316 & 0.189 \\
\hline $\begin{array}{l}\text { Bersonal Belief/ } \\
\text { religion }\end{array}$ & & $11.92 \pm 2.04$ & 1.562 & 0.119 \\
\hline $\begin{array}{l}\text { Overall quality of } \\
\text { life }\end{array}$ & $11.57 \pm 2.88$ & $11.26 \pm 1.93$ & 1.095 & 0.275 \\
\hline
\end{tabular}

Table 4. Comparison of the scores of life quality before and after intervention in the observation group (mean $\pm S D)$.

\begin{tabular}{lllll}
\hline Items & $\begin{array}{l}\text { After } \\
\text { intervention }\end{array}$ & $\begin{array}{l}\text { Before } \\
\text { intervention }\end{array}$ & $\mathbf{t}$ & $\mathbf{P}$ \\
\hline Physiology & $13.34 \pm 2.18$ & $11.81 \pm 3.05$ & 4.998 & 0.000 \\
\hline Psychology & $13.41 \pm 1.85$ & $12.02 \pm 2.91$ & 4.937 & 0.000 \\
\hline Independence & $13.16 \pm 1.96$ & $11.10 \pm 2.92$ & 7.174 & 0.000 \\
\hline Social relations & $12.70 \pm 1.63$ & $11.41 \pm 2.63$ & 5.106 & 0.000 \\
\hline $\begin{array}{l}\text { Environment } \\
\text { Spiritual support/ } \\
\text { belief/ }\end{array}$ & $11.44 \pm 2.13$ & $10.67 \pm 2.41$ & 2.932 & 0.004 \\
\hline \begin{tabular}{l} 
religion \\
\hline
\end{tabular} & & $11.28 \pm 2.59$ & 4.087 & 0.000 \\
\hline
\end{tabular}




Overall quality of $12.96 \pm 2.23 \quad 11.23 \pm 2.18 \quad 6.794 \quad 0.000$

life

Table 5. Comparison of satisfaction rate of nursing care between two groups of patients after intervention (n (\%)).

\begin{tabular}{|c|c|c|c|c|}
\hline Group & Cases & $\begin{array}{l}\text { Satisfactory rate of treatment } \\
\text { during hospitalization }\end{array}$ & $\begin{array}{l}\text { Satisfaction rate of nursing } \\
\text { work during hospitalization }\end{array}$ & $\begin{array}{l}\text { The overall satisfaction rate at } \\
6 \text { months after discharged }\end{array}$ \\
\hline Observation group & 150 & $120(80.00)$ & $130(86.67)$ & $100(66.67)$ \\
\hline Control group & 150 & $98(65.33)$ & $110(73.33)$ & $80(53.33)$ \\
\hline$x^{2}$ & & 8.123 & 8.333 & 5.556 \\
\hline$P$ & & 0.004 & 0.004 & 0.018 \\
\hline
\end{tabular}

\section{Discussion}

Chronic diseases means chronic non-communicable diseases, including coronary heart disease, hypertension, diabetes, chronic respiratory diseases, malignant tumor, characterized by insidious onset, difficulty of cure, incurable and complex etiology, clinical characteristics are high incidence, high morbidity and high mortality. The characteristics of elderly patients with chronic diseases: first, the prevalence of chronic diseases in the elderly is increasing year by year [14]. Second, it is the coexistence of 1-3 kinds of chronic diseases in the elderly. Third, it is the physical and psychological changes of the elderly, elderly patients prone to sense of loss, anxiety, loneliness, depression, despair and other negative emotions [15]. Chronic diseases promote the demand for medical resources in elderly patients [16]. Routine medical assessments and single interventions have significant deficiencies in the assessment of elderly patients with chronic diseases, leading to the emergence of a Comprehensive Geriatric Assessment (CGA) model [17]. Compared with the conventional medical assessment, CGA also includes physical function assessment, psychological evaluation, social and economic assessment, environmental health assessment, quality of life assessment, assessment of common elderly syndrome or problems, and tobacco and alcohol, health products and other evaluation [18]. CGA is a multi-dimensional, interdisciplinary assessment and diagnostic approach, using subjective and objective way to comprehensively judge physical function, mental, social and economic and the elderly functional status and quality of life, has become the focus of elderly medical research in recent years. Research shows that CGA can meet the health needs of older patients, improve the health function of the elderly, reduce medical costs, and in cancer patients, dialysis patients, dementia and emergency recovery patients play an important role $[19,20]$.

The role of nurses in elderly patients with chronic diseases is to provide patient-centered, personalized care. Nurses play an important role in assessing patient's condition, finding problems and integrating CGA recommendations and coordinating follow-up care [21]. According to the results of CGA, nurses can have the care of patients with problems: (1) publicity and education: through the mission video, health talks to strengthen the patient's awareness of the disease, to understand the possible complications of the disease, inform the prevention measures, to develop good habits. (2) psychological care: for patients with bad mood such as anxiety, depression, fear and so on, strengthen communication and implement individualized psychological guidance and intervention. (3) drug care: Patients with poor memory and poor compliance, nurses need to repeatedly inform the correct medication and the time of scientific medication. (4) diet care: for poor diet and malnutrition, we can make a scientific and reasonable diet plan with the nutrition department. (5) exercise care: choose walking, walking, jogging and other appropriate exercise according to the conditions of the patients, increase the body's defense capability. The results showed that there was no significant difference in the control group before and after the intervention in the physiology, psychology, independence, environment, the social relations and the overall quality of life, indicating that no significant change in quality of life after the intervention. The results showed that the observation group after the intervention in the field of physiology, psychology, independence, environment, social relations, overall quality of life were higher than those of the observation group before the intervention, indicating that quality of life was better than before intervention. After the intervention, the satisfaction rate of the observation group and the control group showed that the satisfaction rate and nursing satisfaction of the observation group were significantly higher than those of the control group. From the above results: because of the complexity and specificity of elderly patients, the existing conventional medical care can improve the disease status of patients to a certain extent.

The individualized nursing intervention based on the CGA of the elderly is in line with the modern biological-psychologicalsocial medical model, which can find the potential clinical problems, psychological problems, social demands and economic situation of the elderly patients, pay attention to the individual differences of the patients, satisfy the diverse requirements, make out the patients with personalized nursing plan and feasible treatment measures and the intervention, prevention and health care, can improve the quality of life of patients, and with the approval of patients and their families, improve patient satisfaction. Therefore, the individualized nursing intervention based on CGA is an active exploration and extension of the conventional nursing methods, and explores the direction of the reform direction of the medical 
care model. A number of foreign hospitals have been regarded CGA as a routine treatment [22], but in our country, CGA mostly in the elderly outpatient department, community and other local communities, individualized nursing based on CGA carried out at home and hospital medical staff is very limited, is worth exploring.

In summary, personalized care based on CGA can provide individualized nursing plan for patients with chronic diseases, not only improve the patients' satisfaction with nursing service, improve the quality of life of patients, which is to explore a new mode of nursing, worthy of clinical promotion.

\section{References}

1. Lancet T. Global elderly care in crisis. Lancet 2014; 383: 927-935.

2. Clegg A, Young J, Iliffe S. Frailty in elderly people. Lancet 2013; 381: 752-762.

3. Koroukian SM, Schiltz N, Warner DF. Combinations of chronic conditions, functional imitations, and geriatric syndromes that predict health outcomes. J Gen Intern Med 2016; 31: 630-637.

4. Luo Z. Effect of psychological nursing on psychological state of elderly patients with chronic diseases. China Med Pharm 2016; 32: 521-526.

5. Overcash J, Momeyer MA. Comprehensive geriatric assessment and caring for the older person with cancer. Semin Oncol Nurs 2017; 35: 850-858.

6. Pilotto A, Cella A, Pilotto A. Three decades of comprehensive geriatric assessment: evidence coming from different healthcare settings and specific clinical conditions. J Am Med Dir Assoc 2017; 18: 191-197.

7. Jian JY, Liu Y. The effect of individualized nursing intervention on the treatment and quality of life with elderly chronic obstructive pulmonary disease. China Mod Med 2016; 51: 530-539.

8. Peng LJ, Chen Y, Fei XU. The effect of individualized nursing method guided by Omaha system on elderly patients with coronary heart disease. Nurs Pract Res 2017; 45: 850-858.

9. Case IA. Loewenstein occupational therapy cognitive assessment to evaluate people with addictions. Occup Ther Int 2017; 2017: 1-7.

10. Kakrani VA, Desale AV, Mehta CP. Geriatric depression scale (GDS): a tool for assessment of depression in elderly. J Krishna Inst Med Sci Univ 2015; 4: 24-31.

11. Ito T. Comprehensive physical function assessment in elderly people. Clin Phys Ther 2017; 28: 2572-2579.

12. Sharvanan E, Shravan S, Anand KBS. A cross sectional study on assessment of mini nutritional status and depression status among elderly in the villages of V. Kota Mandal, Chittoor District, A.P. Int J Health Sci Res 2015; 5: 26-31.

13. Yazdani K, Nedjat S, Karimlou M. Developing a shortened quality of life scale from Persian version of the WHOQOL-100 using the Rasch analysis. Iran J Publ Health 2015; 44: 522-534.

14. Mwangi J, Kulane A, Le VH. Chronic diseases among the elderly in a rural Vietnam: prevalence, associated sociodemographic factors and healthcare expenditures. Int $\mathrm{J}$ Equity Health 2015; 14: 1-8.

15. Imahori M, Izumida N, Shirase Y. Effects of preventive long-term care on physical and psychological health status for the elderly: evidence from a salon for the elderly in Abashiri City. Nihon Koshu Eisei Zasshi 2016; 63: 675-682.

16. Ellis G, Gardner M, Tsiachristas A. Comprehensive geriatric assessment for older adults admitted to hospital. Cochr Datab Syst Rev 2017; 24: 890-899.

17. Tuttle CSL, Maier AB. Towards a biological geriatric assessment. Exp Gerontol 2017; 8: 556-565.

18. Tucci A, Martelli M, Rigacci L. Comprehensive geriatric assessment is an essential tool to support treatment decisions in elderly patients with diffuse large B cell lymphoma: a prospective multicenter evaluation on 173 patients by the lymphoma Italian Foundation (FIL). Leuk Lymph 2015; 56: 921-927.

19. Shahrokni A, Kim SJ, Bosl GJ. How we care for an older patient with cancer. J Oncol Pract 2017; 13: 95-102.

20. Pilotto A, Cella A, Pilotto A. Three decades of comprehensive geriatric assessment: evidence coming from different healthcare settings and specific clinical conditions. J Am Med Dir Assoc 2017; 18: 192-197.

21. Ekdahl AW, Sjostrand F, Ehrenberg A. Frailty and comprehensive geriatric assessment organized as CGAward, or CGA-consult, for older adult patients in the acute care setting: A systematic review and meta-analysis. Eur Geriatr Med 2015; 6: 523-540.

22. Mccarthy AL, Cook PS, Yates P. Engineering the fitness of older patients for chemotherapy: an exploration of comprehensive geriatric assessment in practice. Health (London) 2014; 18: 196-212.

\section{*Correspondence to}

Yan-ling $\mathrm{Hu}$

Department of Basic Medicine

Chongqing Three Gorges Medical College

PR China 